\title{
Design of Automatic Greenhouse Environmental Monitoring System
}

\author{
Dong Liang ${ }^{1, a}$ and Limin Shao ${ }^{1, b^{*}}$ \\ ${ }^{1}$ College of Mechanical and Electrical Engineering, Hebei Agricultural University, Baoding, China \\ a1340913757@qq.com, bshaolm@126.com
}

\section{Keywords: Greenhouse; GPRS; Automatic control}

\begin{abstract}
In order to satisfy the planting requirements of vegetables in north cold area of China, an automatic monitoring and controlling system of greenhouse interior environment was developed. By synthetically considering the temperature, humidity, illumination intensity, $\mathrm{CO}_{2}$ concentration, and their mutual influences, the system adopts an environmental regulation method based on set point control to automatically control the internal environment of the greenhouse. The system could provide a long-term stable tool to measure and analyze the environmental information in greenhouses. The test showed that the temperature in greenhouse could be maintained at the needed level while the relative humidity and illumination intensity could be controlled within the suitable scope as well.
\end{abstract}

\section{Introduction}

The greenhouse system is regarded as the innovation of modern agriculture that provides crops with the favorable environmental conditions [1]. A greenhouse is a closed environment that can be actively controlled to improve the crop yields [2]. Therefore, monitoring and regulating the greenhouse environment is very important for growing crops in the greenhouse. There has been a rich literature focusing on the monitor and control problem in the greenhouse [3-6]. In [3], with deployed wireless sensor networks (WSN), the system automatically collect greenhouse environmental information and crop growing information, then transmits the data to a remote database, and uses the Web to monitor the greenhouse. In [4], the Genetic Algorithm (GA) and Particle Swarm Optimization (PSO) algorithm has been introduced into the greenhouse environment monitor system, and the parameter reduction dimension in the greenhouse environmental control model was realized. In [5], a high-level multi-objective optimization method was studied to achieve greenhouse control. In [6], climate simulation was conducted for artificial growth box and greenhouse with temperature gradient, and the crop response under simultaneous/separate effects of temperature, $\mathrm{CO}_{2}$ concentration and water utilization were studied. The system proposed in this paper is designed based on the greenhouse environment information collection and automatic control, aiming to improve the crop growth environment and improve crop quality.

\section{System Architecture}

The greenhouse is an agricultural technology that can change the environment in which plants grow. According to the optimal growth conditions for crop growth, the greenhouse climate is adjusted to meet the needs of plants throughout the year. There are many environmental factors that need to be noticed during the growing process of crops. The main factors affecting the greenhouse environment are temperature and humidity, illumination intensity, and carbon dioxide concentration as the monitoring targets. The greenhouse system consists of micro-controller, sensors net, control module and GPRS-DTU. The overall structure is shown in Fig. 1. The system was structured based on STM32F103ZET6 microprocessor which realizes the collection of greenhouse environmental parameters and the control of the implementing agency. Then, the environment parameter data were sent to the server of monitoring center via GPRS network. Finally, the remote monitoring system can store data and analyze data. 


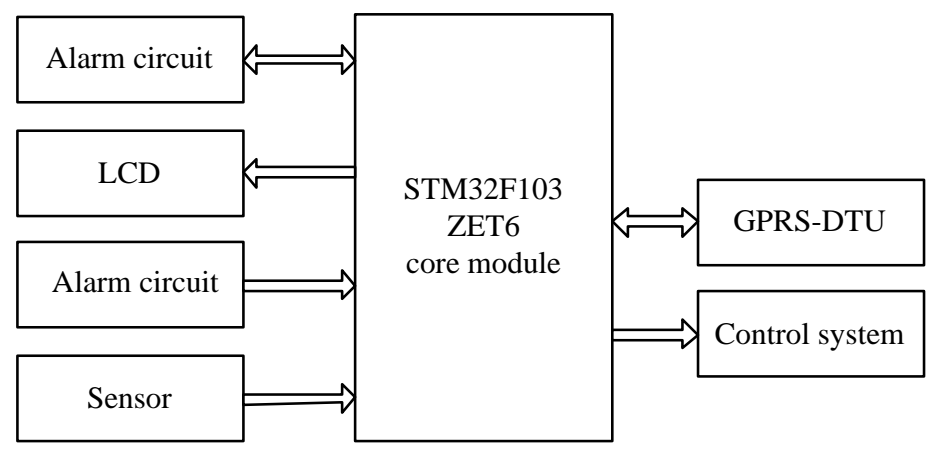

Figure 1. Overall system diagram

\section{Hardware System Design}

Sensor. The sensor is the main part of greenhouse automatic control system. The system mainly implements corresponding control actions based on sensor feedback information. Therefore, the stability, accuracy, and cost performance of the sensor will directly affect the function and cost of the whole system [7-8]. The sensor is encapsulated in the waterproof case of the louver box. Meanwhile, in order to prevent the high humidity environment in the greenhouse from affecting the sensor and measuring circuit, a waterproof and air-permeable treatment is performed on the plastic enclosure. The sensor parameters of the system are shown in Table 1.

Table 1 Sensor parameters

\begin{tabular}{ccccc}
\hline Device name & Model & Working voltage & Measurement range & Accuracy \\
\hline $\begin{array}{c}\text { Temperature and } \\
\text { humidity sensor }\end{array}$ & DWS-T8 & $12 \sim 30[\mathrm{~V}]$ & $-20 \sim+60\left[{ }^{\circ} \mathrm{C}\right]$ & $\pm 0.4\left[{ }^{\circ} \mathrm{C}\right]$ \\
$\begin{array}{c}\text { Illumination sensor } \\
\begin{array}{c}\text { Carbon dioxide } \\
\text { concentration } \\
\text { sensor }\end{array}\end{array}$ & DZD-T4 & $18 \sim 30[\mathrm{~V}]$ & $0 \sim 100[\%]$ & $\pm 3[\%]$ \\
\hline
\end{tabular}

Data Collection. The sensor net consists of microprocessors, sensors and RF module. The structure diagram of data acquisition is shown in Fig. 2. The working process of the data collection is: first, sensors collect information of environment parameters, second, data are sent to the STC12C2052AD processor, and finally data are sent to the STM32F103RCT6 processor through wireless radio frequency module.

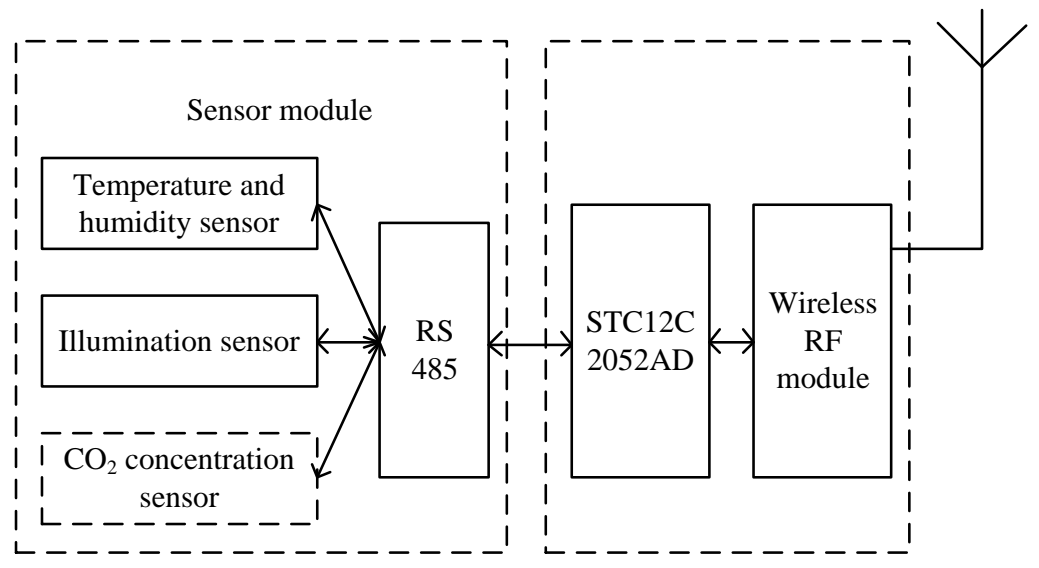

Figure 2. The structure diagram of data acquisition system

Data Transfer. In this system, remote data transmission of environmental parameters is accomplished using GPRS technology [9-11]. GPRS-DTU can provides GPRS data transfer function, 
the built-in TCP/IP protocol stack make it easy to use the TCP/IP protocol easily. It provides two types of operation mode: data transparent mode and the AT command mode. According to the characteristics of this system, the data transparent mode is selected to realize remote data transmission, once the connection is established under transparent mode, the module will be in data mode. All data received from serial port will be treated as data packet to be transferred later, as well all data received from remote server will be sent to serial port directly. This system takes the PC as a server, which connected to the Internet, the IP of PC must be a public network IP address, GPRS module as the client, the MCU control the GPRS module to send a TCP connection request to the server via AT commands, a dynamic IP address will be assigned to the SIM card inside of the module, then the data collected by sensors are sent to the PC through the IP address. Finally we store data into the database for further analysis. The structure diagram of data transmission is shown in Fig. 3.

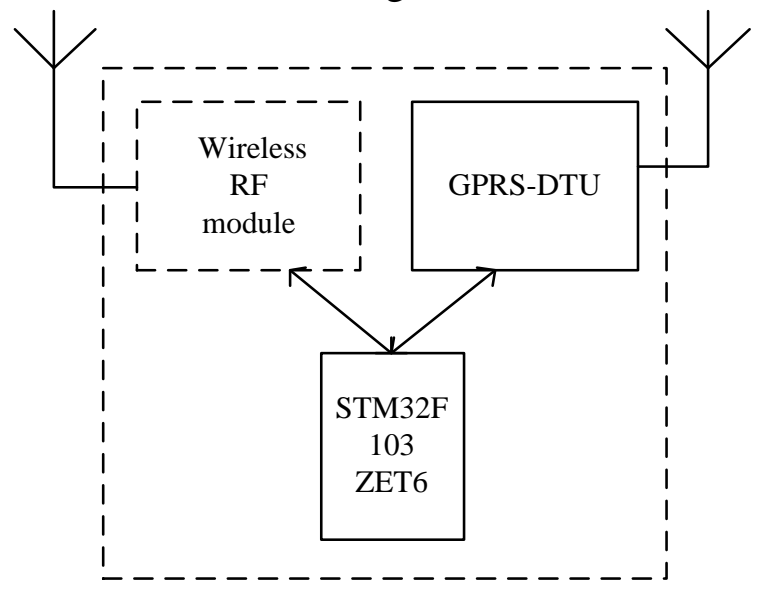

Figure 3. The structure diagram of data transmission system

Microcontroller Control Program Design. This part is mainly divided into the main function control module and the data processing sub-module. The flow charts are shown in Fig. 4. The specific operation flow is as follows:
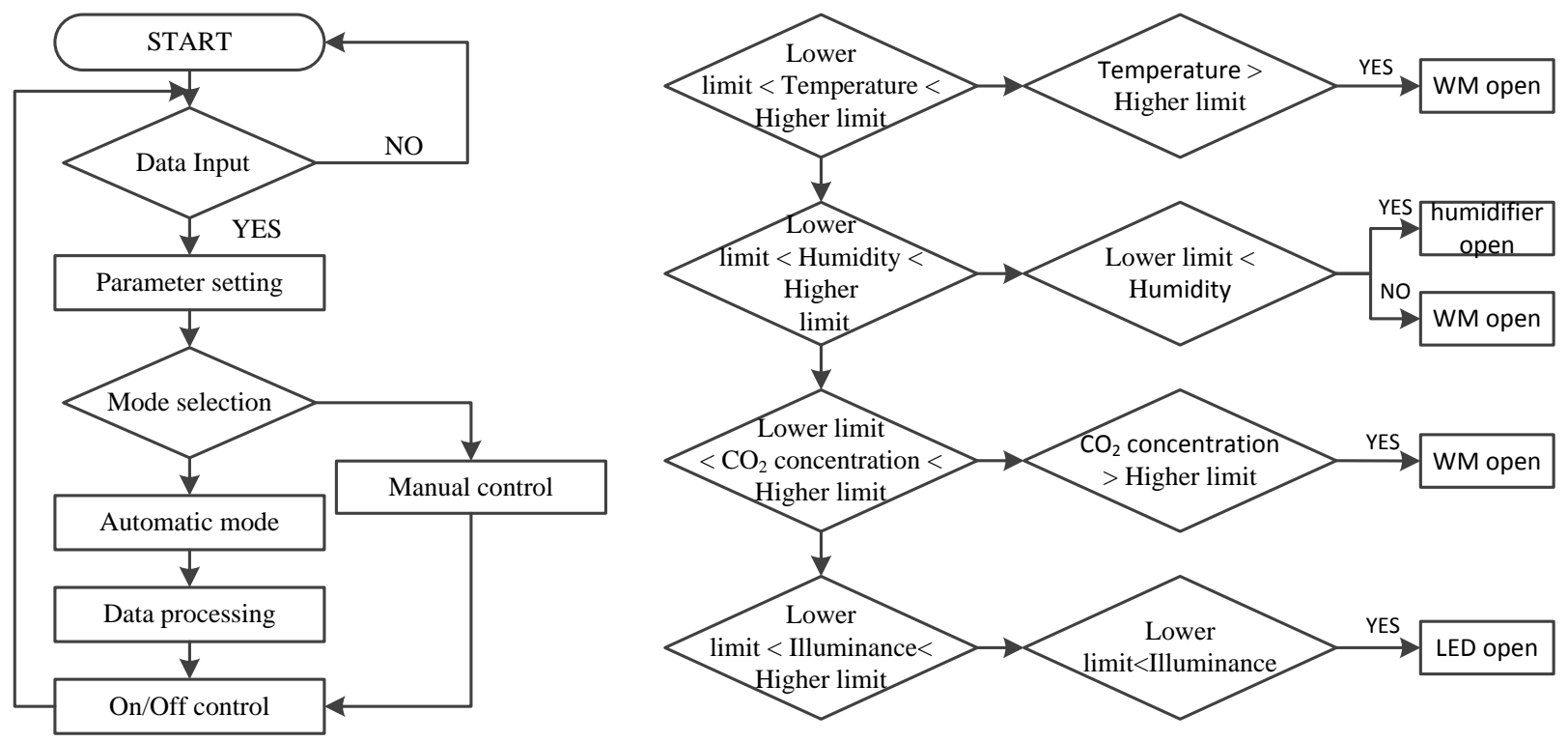

Figure 4. Control program flow chart

(1) Whether to receive data;

(2) Set the parameters of temperature, humidity, illumination intensity and $\mathrm{CO}_{2}$ concentration as required: upper limit value, lower limit value and standard value;

(3) According to the need to choose the control mode: automatic control or manual control;

(4) Calculates the acquisition value of each sensor; 
(5) According to the data collected from the sensor and the set value to determine whether to turn on or off the corresponding device;

(6) The device operation situations and all data in the above total processes are stored in a database.

The Circuit of STM32. The hardware circuit of this system is mainly based on STM32. Since the selected sensor and GPRS-DTU are all RS485 communication methods, STM32 receives data and sends data to the data through USART2. The wiring diagram of partial schematic diagram of STM32, usart 2 circuit, key circuit is shown in Fig. 5. Among them, GPIOC0-3 is connected with 1-4 of the four relays respectively. GPIOPE2-4 and PA0 are 4 keys that control the transformation of high and low level of 4 pins respectively to control the switch of the relay.

Finally, the program of STM32F103 microcontroller is programmed and compiled through MDK5 software environment, and then the file is downloaded to microcontroller through the ST-LINK simulator.
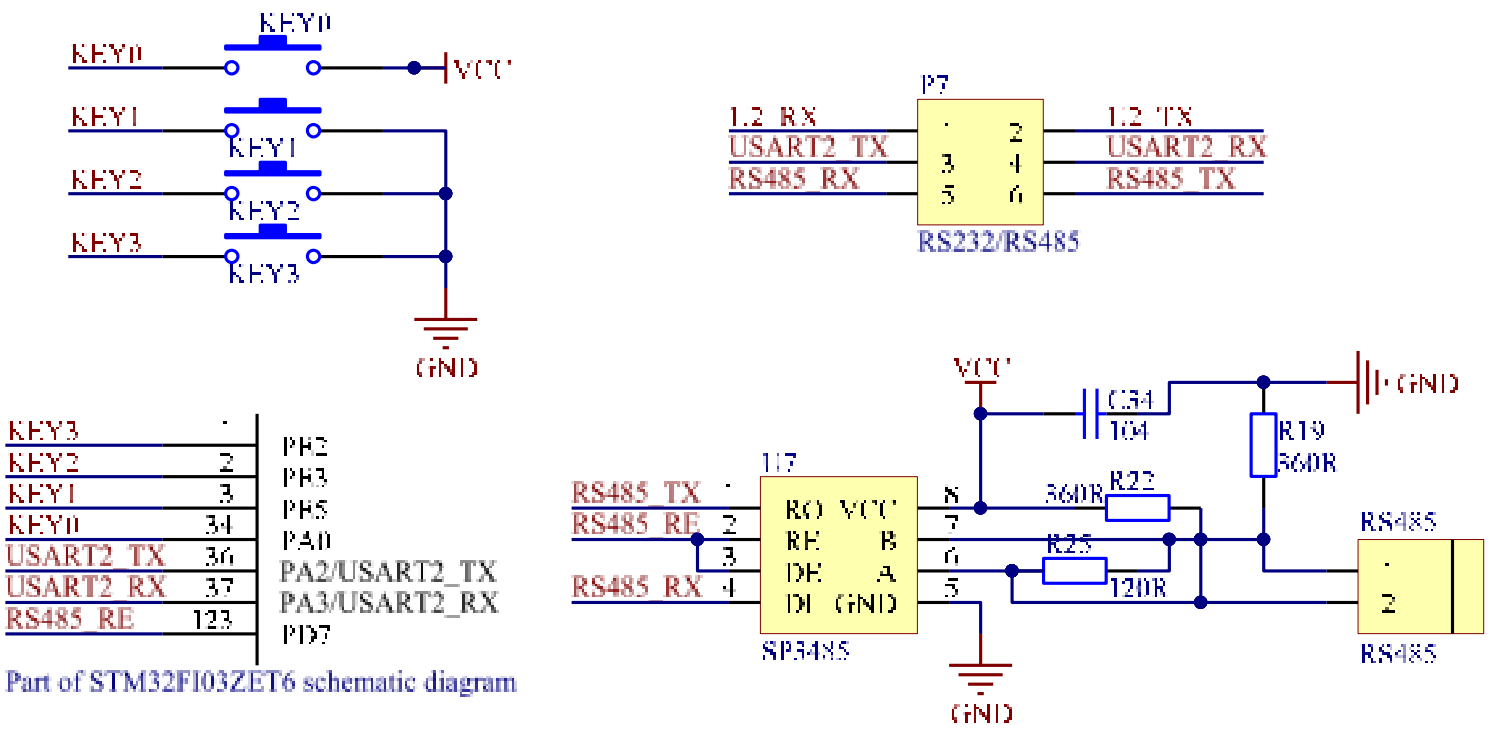

Figure 5. The circuit of STM32

\section{Conclusions}

In this paper, the greenhouse environment monitoring system based on 32-bit processor, wireless RF technology and GPRS is studied, realizing four functions of data collection, data transmission, data storage and data processing. Preliminary experimentations showed that this system met the application requirements of greenhouse monitoring system excellently, and had the advantages of low power consumption, simple structure and strong. Through a large amount of data obtained from the system, the establishment of greenhouse crop growth model that describes the relationship between external variables and plant environment will be the focus of future research.

\section{Acknowledgements}

This study was supported by the Key Research and Development Program of Hebei Province (18227209D, 18227209D-1).

\section{References}

[1] D.A. Gelder, J.A. Dieleman: An Overview of Climate and Crop Yield in Closed Greenhouses, J. Hortic. Sci. Biotechnol, Vol. 87 (2012) No.3, p. 193.

[2] O. Mirabella, M. Brischetto: A Hybrid Wired/Wireless Networking Infrastructure for Greenhouse Management (Pune, India, September 9-10, 2016). Vol. 60, p.154. 
[3] B. Kang, D. Park, K. Cho, et al.: A Study on the Greenhouse Auto Control System Based on Wireless Sensor Network (Hainan Island, China, December 13-15, 2008). p.44.

[4] A Hasni, R Taibi and R Taibi: Optimization of Greenhouse Climate Model Parameters Using Particle Swarm Optimization and Genetic Algorithms, Energy Procedia, Vol. 6 (2011) No.6, p.371.

[5] M Berenguel: Multiobjective Hierarchical Control Architecture for Greenhouse Crop Growth, Automatica, Vol. 48 (2012) No.3, p.490.

[6] E Mashonjowa, F Ronsse and JR Milford: Modelling the Thermal Performance of a Naturally Ventilated Greenhouse in Zimbabwe Using a Dynamic Greenhouse Climate Model, Solar Energy, Vol. 91 (2012) No.3, p.381.

[7] X.Z. Bai, Z.D. Wang, L. Zou, et al.: Collaborative Fusion Estimation over Wireless Sensor Networks for Monitoring $\mathrm{CO}_{2}$ Concentration in a Greenhouse, Information Fusion, Vol. 42 (2012), p.119.

[8] X.H. Li, X. Cheng, K.Yan, et al.: A Monitoring System for Vegetable Greenhouses based on a Wireless Sensor Network, Sensors, Vol. 10 (2010) No.10, p.8963.

[9] J.X. Gao and H.Q. Du: Design of Greenhouse Surveillance System Based on Embedded Web Server Technology, Procedia Engineering, Vol. 23 (2011), p.374.

[10] T Vaithianathan, H.Y. Zhou and J Hauer: Wireless Bi-Directional Data Link for an EEG Recording System Using STM32 (Lisboa, Portugal, June 11-12, 2014), p.314.

[11]H.F. Zhang and W. Kang: Design of the Data Acquisition System Based on STM32, Procedia Computer Science, Vol. 17 (2013), p.222. 